\title{
Gemcitabine resistance in triple-negative breast cancer cells can be reverted by Drosophila melanogaster deoxyribonucleoside kinase in the nucleus or cytosol
}

\author{
YAN ZHAO $^{1}$, HAIYANG JIANG ${ }^{1}$, MING GU ${ }^{1}$, CONG ZU ${ }^{2}$ and XINYU ZHENG ${ }^{1,2}$ \\ ${ }^{1}$ Department of Breast Surgery, The First Affiliated Hospital, China Medical University, Shenyang, Liaoning 110001; \\ ${ }^{2}$ Lab 1, Cancer Institute, The First Affiliated Hospital, China Medical University, Shenyang, Liaoning 110001, P.R. China
}

Received October 20, 2019; Accepted July 28, 2020

DOI: 10.3892/ol.2020.12109

\begin{abstract}
The development of drug resistance to chemotherapeutic agents has consistently presented a challenge in terms of the treatment of patients with triple-negative breast cancer (TNBC). In the present study, gemcitabine (dFdC)-resistant TNBC cells were established, and the effects of lentivirus-deoxyribonucleoside kinase (dNK) and a mutated form of $\mathrm{dNK}$ (lentivirus-dNKmut) on reversing the acquired drug resistance in $\mathrm{dFdC}$-resistant TNBC cells were explored. Quantitative PCR and western blotting experiment results suggested that Drosophila melanogaster (Dm)-dNK was stably expressed in the lentivirus-infected MDA-MB-231 and MDA-MB-231R cells in the nucleus or cytosol, and autoradiography experiments revealed similar levels of enzymatic activity in the cells expressing $\mathrm{dNK}$ or dNKmut. In vitro cytotoxicity assay revealed that the $\mathrm{IC}_{50}$ values of $\mathrm{dFdC}$ were decreased 30 50-fold in the dFdC-resistant MDA-MB-231 cells following lentiviral transfection with dNK or dNKmut, and this effect was associated with a significantly increased rate of apoptosis compared with the cells transfected with the negative control lentivirus. In conclusion, Dm-dNK in the nucleus or cytosol may be a potential candidate for reversing acquired $\mathrm{dFdC}$ resistance in TNBC cells, which may form the basis of novel strategies for the treatment of patients with drug-resistant TNBC.
\end{abstract}

\section{Introduction}

Triple-negative breast cancer (TNBC) accounts for 15-20\% of all breast carcinomas and is associated with an aggressive disease progression and a high risk of relapse $(1,2)$.

Correspondence to: Dr Xinyu Zheng, Department of Breast Surgery, The First Affiliated Hospital, China Medical University, 155 North Nanjing Street, Shenyang, Liaoning 110001, P.R. China E-mail: xyzheng@cmu.edu.cn

Key words: Drosophila melanogaster deoxyribonucleoside kinase, suicide gene, nucleoside analogue, drug resistance, gemcitabine, triple-negative breast cancer
The median survival time of relapsed patients with TNBC varies between 12 and 24 months (3). TNBC is not efficiently treated by the currently available therapeutic regimens; this may be attributed to the lack of estrogen, progesterone and Erb-B2 receptor tyrosine kinase 2 (ERBB2) receptors $(4,5)$. Chemotherapy remains the primary systemic treatment, and the poor prognosis of TNBC is often ascribed to resistance to chemotherapeutic agents. After repeated cycles of chemotherapy, enhanced tumor resistance and severe side effects resulting from these agents worsen the clinical outcome, often leading to therapeutic failure (6).

In recent years, various lines of research have been performed that aimed to bypass drug resistance and improve the sensitivity to chemotherapeutic agents in cancer cells $(7,8)$. The underlying mechanisms are often complicated, such as reducing the effective drug concentration in cells, establishing abnormalities in drug targets and altering the regulation of apoptosis (9). Various strategies, including RNA silencing, nanopreparations, co-administration of two or more strategies, novel cytotoxic agents and regulation of apoptosis, have been developed to overcome drug resistance in cancer cells $(10,11)$.

Gemcitabine (2',2'-difluorodeoxycytidine; $\mathrm{dFdC}$ ) has been evaluated for its efficacy in the treatment of TNBC in a number of clinical trials and has been established as one of the most efficient chemotherapeutic drugs for various types of cancer in clinical practice $(12,13) . \mathrm{dFdC}$ is taken up into the cells by human equilibrative nucleoside transporters and human concentrative nucleoside transporters $(14,15)$. Once inside the cell, $\mathrm{dFdC}$ is phosphorylated by deoxycytidine kinase $(\mathrm{dCK})$ to its monophosphorylated form, and subsequently by nucleotide kinases to its active metabolites, $\mathrm{dFdC}$ diphosphate (dFdCDP) and $\mathrm{dFdC}$ triphosphate (dFdCTP) (16). dFdCDP is an effective inhibitor of ribonucleotide diphosphate reductases including ribonucleoside-diphosphate reductase large subunit (RRM1), and resistance to $\mathrm{dFdC}$ is associated with increased expression of ribonucleotide reductase (17).

The phosphorylation induced by $\mathrm{dFdC}$ is the main rate-limiting step of the anticancer effect of $\mathrm{dFdC}$ (18); on the other hand, $\mathrm{dFdC}$ can be deactivated to its main metabolite 2',2'-difluorodeoxyuridine (dFdU) by cytidine deaminase (CDA) (19). The cytotoxicity of $\mathrm{dFdC}$ is mainly associated with the cellular accumulation of dFdCTP (16). 
$\mathrm{dFdCTP}$ is incorporated into DNA, causing masked chain termination by inducing a $G_{0} / G_{1}$ and $S$-phase arrest in the cell cycle, which triggers apoptosis (20). In addition, dFdC decreases cellular deoxynucleotide (dNTP) pools and competes with them for incorporation into DNA, which, coupled with a decreased feedback inhibition of $\mathrm{dCK}$, leads to an enhanced incorporation of $\mathrm{dFdC}$ into DNA (18).

Various therapeutic approaches have been proposed to overcome drug resistance induced by nucleoside kinase deficiency (21-23). Previously published work from our laboratory has demonstrated that a multisubstrate deoxyribonucleoside kinase of Drosophila melanogaster (Dm-dNK) may be a potential candidate suicide gene, and its effectiveness has been investigated in a number of tumor cell lines, including MDA-MB-231 (24), using viral systems (retrovirus-, adenovirus- and lentivirus-based vectors) combined with prodrugs such as araT, araC, gemcitabine and bromovinyldeoxyuridine (BVDU) (25-31). The effectiveness of Dm-dNK has been demonstrated to be due to its broad substrate specificity regarding both purine and pyrimidine nucleoside analog phosphorylation and a higher catalytic rate compared with that of previously studied nucleoside kinases (32-34).

Previous studies have demonstrated that dCK-deficient cell lines display a $\mathrm{dFdC}$-resistant phenotype $(35,36)$. Therefore, the hypothesis of the present study was that transfection with Dm-dNK may reverse the resistance to $\mathrm{dFdC}$ in TNBC cells. The present study aimed to develop a dFdC-resistant breast cancer MDA-MB-231 cell model to explore whether Dm-dNK may reverse $\mathrm{dFdC}$ resistance in TNBC and to determine the underlying mechanisms.

\section{Materials and methods}

Lentiviral packaging and titration. The basic plasmid was established has been previously described (31,37). Briefly, a three-plasmid system and lentiviral vectors were co-transfected into the packaging cells (the 293 cell line; $5 \times 10^{6}$ cells $/ 100-\mathrm{mm}$ dish cultured for $24 \mathrm{~h}$ until the cell confluence rate was $70-80 \%$ ) through standard transient transfection using Lipofectamine ${ }^{\circledR}$ 2000 (Thermo Fisher Scientific, Inc.) according to the manufacturer's protocol. The rate of plasmid:vector:cells was 1:1:1. The medium was collected $48 \mathrm{~h}$ post-transfection and filtered through a $0.45-\mu \mathrm{m}$ filter and diluted 2-fold with fresh medium, and then repeatedly collected three times at 24-h intervals. Subsequently, PCR was used to amplify the DNA fragment of Dm-dNK-3Flag and Dm-dNKmut-3Flag, and the fragment was then ligated into a PGC-FU plasmid (Shanghai GeneChem Co., Ltd.), which was composed of a 5'-long terminal repeat, a cytomegalovirus promoter and a multiple cloning site in the presence of a green fluorescent protein (GFP) sequence. Virus-producing cells were collected by ultracentrifugation $\left(4^{\circ} \mathrm{C}, 120,000 \mathrm{x} \mathrm{g}\right.$ for $2 \mathrm{~h}$ ) to collect the recombinant lentivirus, followed by PCR identification. These lentiviruses were termed Lv-dNK and Lv-dNKmut. Lentiviral infectivity among cell lines was determined by dNK-GFP and dNKmut-GFP. To improve the infection efficiency, all cell lines were transduced $\left(37^{\circ} \mathrm{C}\right.$ for $24 \mathrm{~h}$ repeated three times) with lentivirus in DMEM supplemented with $6 \mu \mathrm{g} / \mathrm{ml}$ polybrene (Merck KGaA). The virus titer was quantified based on the number of GFP-positive cells and the infectious dose of the recombinant virus with 10-fold serial dilution. The cell nuclei were counterstained with 4',6-diamidino-2-phenylindole (DAPI). GFP and DAPI fluorescence was observed using a Nikon Eclipse E600 microscope (Nikon Corporation) equipped with a SPOT RT digital camera (Diagnostic Instruments, Inc.) at x400 magnification in 5-6 fields per sample.

Cell culture and establishment of the dFdC-resistant cell line. 293 and MDA-MB-231 cells were obtained from The Cell Bank of Type Culture Collection of the Chinese Academy of Sciences. HEK293 and MDA-MB-231 cells were maintained in high-glucose Dulbecco's modified Eagle's medium (DMEM) supplemented with $10 \%$ fetal bovine serum (FBS), $100 \mathrm{U} / \mathrm{ml}$ penicillin and $100 \mathrm{U} / \mathrm{ml}$ streptomycin at $37^{\circ} \mathrm{C}$ in a humidified atmosphere containing $5 \% \mathrm{CO}_{2}$. The modified resistant strain of MDA-MB-231 was developed as previously described (38). Briefly, MDA-MB-231 cells were maintained in DMEM and exposed to $\mathrm{dFdC}$ at an initial concentration of $1.0 \mu \mathrm{M}$ and repeatedly cultured with increasing concentrations of $\mathrm{dFdC}$ at 1.5-2-fold increments including $\mathrm{dFdC}$-free intervals in order to allow the surviving cells to recover. MDA-MB-231 cells were seeded at a density of $3 \times 10^{3}$ cells/100-mm dish and subcultured every 14 days with increasing dosage of $\mathrm{dFdC}$ treatment from day 7 to day 12 . This protocol was repeated twice. After 28 days, the cells were seeded at a density of $6 \times 10^{5}$ cells $/ 100-\mathrm{mm}$ dish and treated with $\mathrm{dFdC}$ after $24 \mathrm{~h}$. After another $72 \mathrm{~h}$, the cells were subcultured and cultured in $\mathrm{dFdC}$-free medium for $96 \mathrm{~h}$ to allow the surviving cells to recover. This protocol was repeated five times. The final concentration was $80 \mu \mathrm{M}$. Chemoresistant MDA-MB-231 cells were challenged with $\mathrm{dFdC}$ for 6 months. Prior to the experiments, chemoresistant MDA-MB-231 cells were seeded into to the drug-free medium for 2 weeks, and these cells were termed MDA-MB-231R cells.

Western blotting analysis. MDA-MB-231 and MDA-MB-231R cells were harvested at $72 \mathrm{~h}$ following lentiviral infection, and total proteins were extracted using a lysis buffer [50 mM HEPES (pH 7.4), 250 mM NaCl, 1 mM NaF, 1 mM EDTA, $1 \%$ Triton X-100 and $1 \mathrm{mM}$ DTT] supplemented with protease inhibitors PMSF (cat. no. 8553S; Cell Signaling Technology, Inc.). The concentration of the extracted protein was measured using a BCA protein assay (Nanjing KeyGen Biotech Co., Ltd.). Equal amounts $(20 \mu \mathrm{g})$ of the proteins were subjected to SDS-PAGE (10\% gels) and electro-transferred to PVDF membranes (EMD Millipore). The blots were blocked in Tris-buffered saline (TBS) containing $0.1 \%$ Tween-20 and 5\% nonfat milk at room temperature for $2 \mathrm{~h}$, followed by incubation with antibodies against Flag (1:1,000; cat. no. ab205606; Abcam), dCK (1:5,000; cat. no. ab151966; Abcam), CDA (1:300; cat. no. SAB1300717; Merck KGaA), P-gp (1:2,000; cat. no. ab170904; Abcam) and $\beta$-actin (1:500; cat. no. sc47778; Santa Cruz Biotechnology, Inc.) at $4^{\circ} \mathrm{C}$ overnight. Subsequently, the blots were washed with TBS containing $0.1 \%$ Tween-20, followed by incubation with an appropriate specific secondary antibody: Mouse anti-rabbit IgG-HRP (1:5,000; cat. no. sc2357; Santa Cruz Biotechnology, Inc.) or goat anti-mouse IgG-HRP (1:4,000; cat. no. sc2005; Santa Cruz Biotechnology, Inc.) at room temperature for $2 \mathrm{~h}$. The immunoreactive bands were visualized with ECL western 
blotting substrate (Thermo Fisher Scientific, Inc.), and the protein expression was detected using the ChemiDoc ${ }^{\mathrm{TM}} \mathrm{XRS}+$ Imaging System (Bio-Rad Laboratories, Inc.). The relative band intensities were estimated using ImageJ 1.48 software (National Institutes of Health). $\beta$-actin was used as an internal loading control.

Reverse transcription-quantitative PCR (RT-qPCR). Total RNA was extracted from the MDA-MB-231 and MDA-MB-231R cells using the AP-MN-MS-RNA-50 RNA extraction kit (Axygen; Corning, Inc.). RT was performed using a PrimeScript RT reagent kit (Takara Biotechnology Co., Ltd.) according to the manufacturer's instructions. PCR amplification of the cDNA was performed in a $25 \mathrm{ml}$ mixture containing $2 \mathrm{ml}$ template cDNA, $1 \mathrm{ml}$ each forward and reverse primer $(10 \mu \mathrm{mol} / \mathrm{l})$ and $12.5 \mathrm{ml} \mathrm{SYBR}{ }^{\circledR}$ Premix Ex Taq II (Takara Biotechnology Co., Ltd.). GAPDH was used as an endogenous control. The primers used were as follows: Dm-dNK forward, 5'-ATGAGTTGCACGAGGACTGG-3' and reverse, 5'-CTGGTACTCGGTGCCAATGT-3'; and GAPDH forward, 5'-ACAGTCCATGCCATCACTGCC-3' and reverse, 5'-GCCTGCTTCACCACCTTCTTG-3'. The thermocycling conditions included an initial incubation at $95^{\circ} \mathrm{C}$ for $30 \mathrm{sec}$, followed by 40 cycles of denaturation at $95^{\circ} \mathrm{C}$ for $5 \mathrm{sec}$ and annealing at $60^{\circ} \mathrm{C}$ for $30 \mathrm{sec}$. Each experiment was performed three times. The target gene levels were normalized to that of GAPDH as the housekeeping gene. Relative expression levels were calculated according to the $2^{-\Delta \Delta \mathrm{Cq}}$ method (39).

Enzyme activity assays. Cellular proteins were extracted using a previously established protocol (40) at $72 \mathrm{~h}$ post-infection with Lv-dNK or Lv-dNKmut at a multiplicity of infection (MOI) of 10. The activity of Dm-dNKmut was determined using a 35-ml reaction mixture comprising $50 \mathrm{mM}$ Tris- $\mathrm{HCl}(\mathrm{pH} 7.6)$, $100 \mathrm{mM} \mathrm{KCl}, 2 \mathrm{mM}$ DTT, $15 \mathrm{mM} \mathrm{NaF}, 5 \mathrm{mM} \mathrm{MgCl} 2,5 \mathrm{mM}$ ATP, $0.5 \mathrm{mg} / \mathrm{ml}$ bovine serum albumin (GBCBIO Technologies, Inc.) and $0.6 \mathrm{mg}$ protein extract. For these analyses, aliquots of the samples, in which $2.5 \mathrm{mM}$ [methyl $-{ }^{3} \mathrm{H}$ ] deoxythymidine (dThd; Moravek, Inc.) was mixed with equivalent amounts of unlabeled substrates, were spotted onto Whatman DE-81 filter paper discs following incubation at $37^{\circ} \mathrm{C}$ for 10,20 and $30 \mathrm{~min}$. Subsequently, the paper discs were dried for $1 \mathrm{~h}$, washed three times with $5 \mathrm{mM}$ ammonium formate, the elution of nucleoside monophosphates was performed using $0.5 \mathrm{M} \mathrm{KCl}$, and the radioactivity was subsequently determined using a scintillation counter.

Cell viability and 3-(4,5-dimethylthiazol-2-yl)-2,5-diphenyltetrazolium bromide (MTT) assay. The viability of MDA-MB-231 and MDA-MB-231R cells was determined using the MTT assay. Briefly, cells were seeded in 96-well plates at a density of $10^{4}$ cells/well and treated with graded concentrations of $\mathrm{dFdC}$ from 0.0001 to $100 \mu \mathrm{M}$ for $48 \mathrm{~h}$ following infection with Lv-dNK or Lv-dNKmut. Subsequently, the medium was replaced with fresh medium (DMEM containing 10\% FBS), and $20 \mu \mathrm{l}$ MTT reagent (5 mg/ml; Promega Corporation) was added to each well, followed by incubation for $4 \mathrm{~h}$. The formazan products were dissolved by $200 \mu 199 \%$ dimethylsulfoxide (DMSO) for $10 \mathrm{~min}$. The absorbance was examined by an enzyme immunoassay instrument at a wavelength of $570 \mathrm{~nm}$. Each experiment was performed in triplicate.
Toxicity analysis with trypan blue dye exclusion assay. Cytotoxicity was evaluated using the trypan blue dye exclusion assay as previously described by Fraser et al (41). Each experiment was performed in a $35-\mathrm{mm}$ tissue culture dish (three dishes per experimental condition) with $3 \times 10^{4}$ cells per dish with $1 \mathrm{ml}$ high-glucose DMEM with $10 \%$ FBS was added. The plates were incubated overnight in the humidified incubator at $37^{\circ} \mathrm{C}$ to allow the cells to settle. The cells were subsequently infected with Dm-dNK, Dm-dNKmut or the empty lentiviral vector at an MOI of 1 . Various concentrations of $\mathrm{dFdC}(0.01$, $0.1,1,10$ and $100 \mu \mathrm{M}$ ) were subsequently added, and the plates were incubated at $37^{\circ} \mathrm{C}$ for a further $24 \mathrm{~h}$. Then, the medium was removed, followed by the addition of $0.25 \mathrm{ml}$ trypan blue dye diluted in $0.8 \mathrm{ml}$ medium. After 10 -min incubation at $37^{\circ} \mathrm{C}$ in the dark, the diluted trypan blue was removed, and 30 fields of view with at least 20 cells in each field were captured under a microscope and counted using ImageJ 1.48 software (National Institutes of Health).

Apoptosis assay. Induction of apoptosis was analyzed by flow cytometry. Briefly, MDA-MB-231 and MDA-MB-231R cells were seeded in 6 -well plates at $2 \times 10^{5}$ cells/well and cultured for $24 \mathrm{~h}$, followed by transduction with lentiviral vectors, Lv-dNK or Lv-dNKmut at MOI of 10. Two days later, $\mathrm{dFdC}(1 \mu \mathrm{M})$ was added for $24 \mathrm{~h}$, and the apoptosis assays were performed using an Annexin V-FITC kit (cat. no. KGA105; Nanjing Keygen Biotech Co., Ltd.) according to the manufacturer's instructions. The ratio of early and late apoptosis was assessed by a FACScan flow cytometer equipped with CELLQUEST and ModFITLT for Mac V1.01 software (Becton-Dickinson and Company).

Statistical analysis. Data are presented as the means \pm SD. Data were analyzed using SPSS 23.0 (IBM Corp.). Differences between two groups were evaluated using unpaired Student's t-test, whereas one- and two-way ANOVA with Bonferroni post hoc test was performed for multiple comparisons. $\mathrm{P}<0.05$ was considered to indicate a statistically significant value.

\section{Results}

Establishment of the drug-resistant breast cancer cell line. Parental cells MDA-MB-231 were continuously challenged with $\mathrm{dFdC}$ for 6 months to generate $\mathrm{dFdC}$-resistant clones. As presented in Fig. 1A, the MDA-MB-231R cells were less sensitive to $\mathrm{dFdC}$ compared with the MDA-MB-231 cells. The mean $\mathrm{IC}_{50}$ values of MDA-MB-231R and MDA-MB-231 were 53.72 and $4.077 \mu \mathrm{M}$, respectively $(\mathrm{P}<0.001)$, exhibiting an $\sim 13$-fold increase in the MDA-MB-231R cells compared with the parental MDA-MB-231 cells. Subsequently, a concentration of $1 \mu \mathrm{M}$ was selected for further experiments. Fig. 1B demonstrates that the cell viability was significantly inhibited in the MDA-MB-231R cells compared with that in the MDA-MB-231 cells after 48-h treatment with $\mathrm{dFdC}$. Furthermore, the difference in cell proliferation between the two cell types increased over time.

The results also demonstrated that the levels of $\mathrm{P}$ glycoprotein (P-gp) were increased in MDA-MB-231R cells compared with those in the parental MDA-MB-231 cells (Fig. 1C). Subsequently, the protein expression levels of $\mathrm{dCK}$ and CDA 

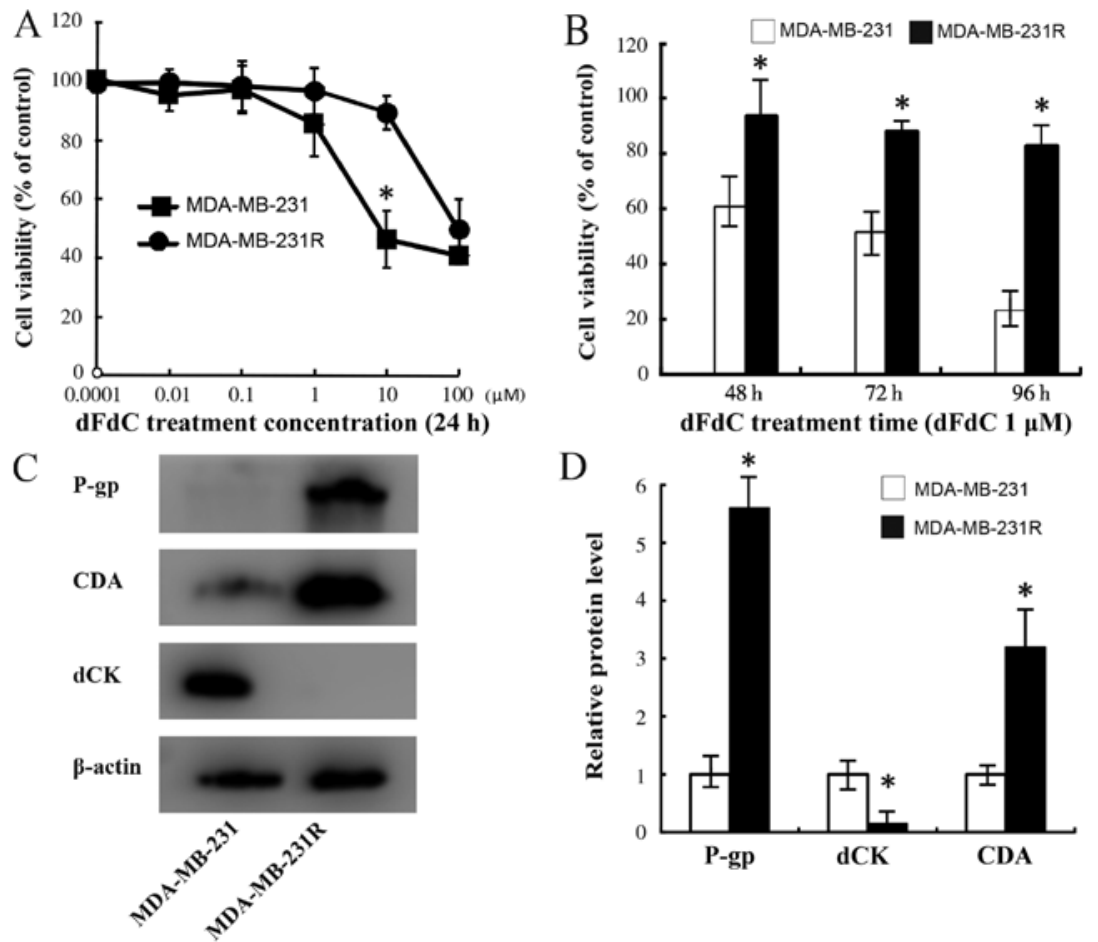

Figure 1. Generation and identification of dFdC-resistant cells. (A) MDA-MB-231 and MDA-MB-231R cells were exposed to various concentrations of dFdC $(0.0001,0.01,0.1,1,10$ and $100 \mu \mathrm{M})$. Cell viability was examined using the MTT assay. The viability of MDA-MB-231R cells was less inhibited compared with that of MDA-MB-231 cells treated with $10 \mu \mathrm{M} \mathrm{dFdC}$. (B) Cells were exposed to $1.0 \mu \mathrm{M} \mathrm{dFdC} \mathrm{for} 48,72 \mathrm{or} 96 \mathrm{~h}$. The viability of MDA-MB-231R cells was less inhibited compared with that in MDA-MB-231 cells after $48 \mathrm{~h}$, and this difference increased over time. (C and D) The protein levels of P-gp, dCK and CDA in MDA-MB-231 and MDA-MB-231R cells were assessed by western blotting analysis. $\beta$-actin was used as an internal control. ${ }^{*} \mathrm{P}<0.05$ vs. MDA-MB-231. $\mathrm{dFdC}$, gemcitabine; dCK, 2',2'deoxycytidine kinase; CDA, cytidine deaminase; P-gp, P-glycoprotein.

were evaluated and were demonstrated to be associated with the $\mathrm{dFdC}$ resistance of MDA-MB-231R cells; the protein expression level of dCK was decreased, whereas that of CDA increased in the MDA-MB-231R cells compared with their parental cells (Fig. 1C and D), which was consistent with the results of previous studies $(23,42)$, suggesting that the $\mathrm{dFdC}$-resistant cell line was successfully established.

Assessment of the enzyme activity of Dm-dNK in the primary and drug-resistant breast cancer cell lines. In order to visualize the subcellular localization of the recombinant enzymes in vivo, the proteins were fused to GFP. MDA-MB-231 and MDA-MB-231R cells were transfected with the recombinant lentiviral vectors. Both cell lines were stably transfected, and green fluorescence was observed in $80-90 \%$ of the cells. Fluorescence in the nucleus was observed in cells transfected with the vector encoding dNK-GFP, whereas it was predominantly detected in the cytosol in cells transfected with the vector encoding dNKmut-GFP (Fig. S1). RT-qPCR and western blotting analysis demonstrated similar mRNA and protein expression levels of dNK-GFP and dNKmut-GFP in the MDA-MB-231 and MDA-MB-231R cells, irrespective of the subcellular localization (Fig. 2A-C).

In the present study, the levels of dThd phosphorylation in protein extracts of MDA-MB-231 and MDA-MB-231R cells were also evaluated to assess the enzymatic activity of the Dm-dNK proteins, as presented in Fig. 2D. The dThd kinase activity was increased 50-fold in MDA-MB-231 and MDA-MB-231R cells expressing nuclear Lv-dNK or cytosolic Lv-dNKmut compared with that in the cells transduced with lentiviral vectors. No significant differences in Dm-dNK activity were detected between the MDA-MB-231 and MDA-MB-231R cells.

Dm-dNK restores $T N B C$ cell sensitivity to $d F d C$. The $\mathrm{dFdC}$ resistance of MDA-MB-231R cells was observed to be reversed following transfection with $\mathrm{Lv}$-dNK or $\mathrm{Lv}$-dNKmut at the MOI of 1 (Fig. 3A and B). The $\mathrm{IC}_{50}$ values for $\mathrm{dFdC}$ in MDA-MB-231R cells expressing Dm-dNK or dNKmut were 1.14 and $1.62 \mu \mathrm{M}$, respectively; The $\mathrm{IC}_{50}$ values for $\mathrm{dFdC}$ in MDA-MB-231 cells expressing dNK or dNKmut were 0.81 and $0.78 \mu \mathrm{M}$, respectively. These values were $\sim 30$-fold lower compared with those in the Lv group, irrespective of the protein localization (Fig. 3A and B), indicating that no differences in sensitivity to nucleoside analogs were observed between cells expressing nuclear and cytosolic Dm-dNK.

To gain further insights into the anticancer potential of Lv-dNK and Lv-dNKmut, apoptosis assay was performed in chemo-resistant MDA-MB-231R cells. As presented in Fig. $4 \mathrm{~A}$ and $\mathrm{B}$, following $24-\mathrm{h}$ exposure to $1 \mu \mathrm{M} \mathrm{dFdC}$, a significant increase in the apoptotic rate was observed in the MDA-MB-231R cells transfected with Lv-dNK and LV-dNKmut compared with that in the control cells transduced with lentiviral vectors. The apoptotic rate in the control cells was 2-3\%, whereas those in the Lv-dNK- and Lv-dNKmut-transfected cells were 19.7 and $14.2 \%$, respectively. In addition, P-gp expression levels were partly decreased following transfection with Lv-dNK or Lv-dNKmut compared with the empty lentivirus group in MDA-MB-231R cells 

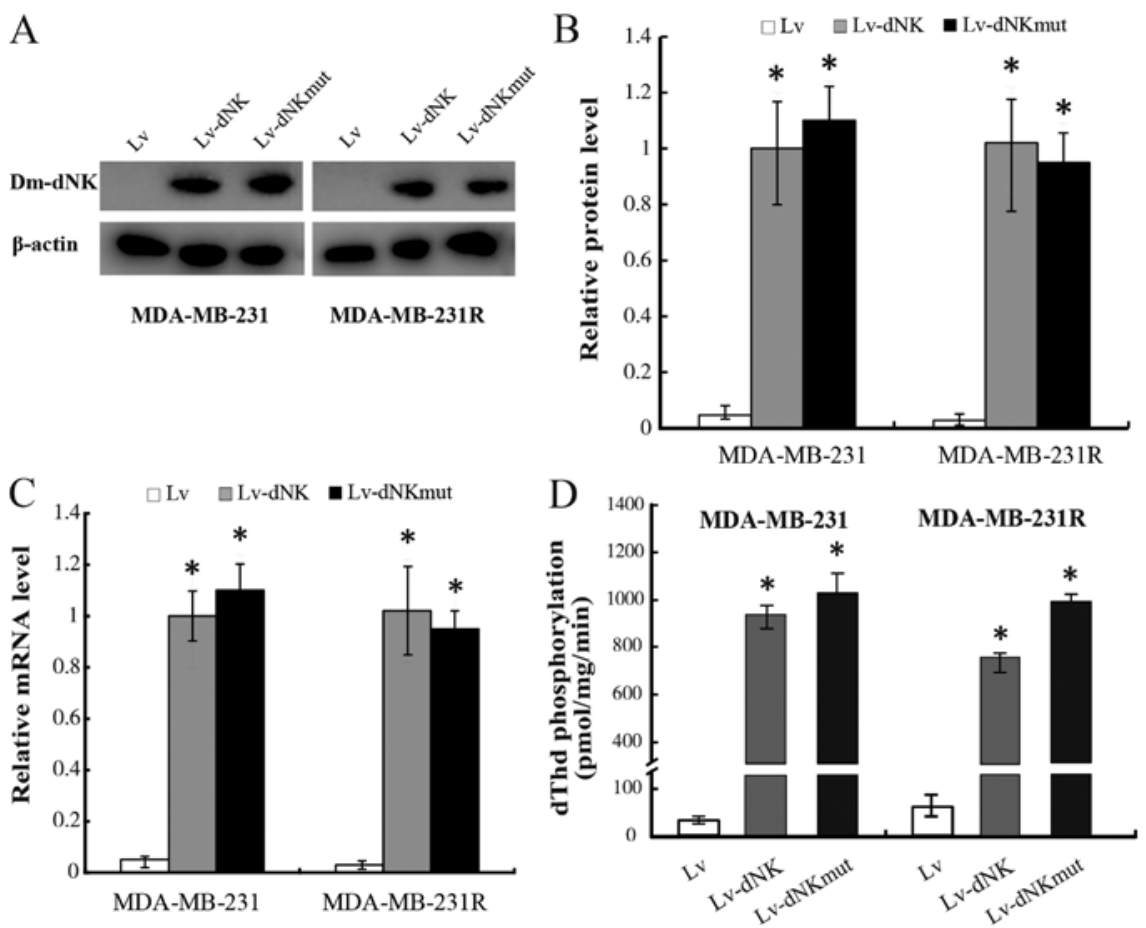

Figure 2. Expression and enzyme activity levels of dNK and dNKmut in transduced MDA-MB-231 and MDA-MB-231R cells. (A and B) Western blotting analysis of cell protein extracts using anti-Flag antibodies to detect dNK-GFP and dNKmut-GFP in MDA-MB-231 and MDA-MB-231R cells. $\beta$-actin was used as an internal control. (C) Quantitative PCR was used to evaluate the mRNA level of dNK and dNKmut in MDA-MB-231 and MDA-MB-231R cells transfected with Dm-dNK or Dm-dNKmut. (D) Dm-dNK activity in crude extracts of MDA-MB-231 and MDA-MB-231R cells transduced with or without Dm-dNK or Dm-dNKmut was determined by dThd phosphorylation. "P $<0.05$ vs. Lv. dThd, deoxythymidine; Dm-dNK, Drosophila melanogaster deoxyribonucleoside kinase; dNKmut, Dm-dNK mutant; Lv, lentivirus.

(Fig. 4C and D). These results revealed that the cell apoptotic rate of MDA-MB-231R cells was significantly increased due to active drug conversion from the prodrug.

\section{Discussion}

Resistance to anticancer drugs is a crucial problem that limits the effectiveness of chemotherapy regimens (43). Resistance generally develops with long-term exposure to the drug (44). In the present study, $\mathrm{dFdC}$ resistance was established in MDA-MB-231 cells through long-term treatment with $\mathrm{dFdC}$, which was verified by MTT assay. Although the emergence of drug resistance has been associated with multiple molecular mechanisms, MDR1, which actively transports toxins out of the cells, has been strongly associated with the development of resistance to various chemotherapeutic agents (45). In the dFdC-resistant MDA-MB-231R cells established in the present study, expression of the MDR1-encoded P-gp was observed, but this was not the case for the MDA-MB-231 cells.

$\mathrm{dCK}$ is essential for the phosphorylation of $\mathrm{dFdC}$, and CDA catalyzes the degradation of dFdC $(18,19)$. dCK and CDA levels have been demonstrated to be significantly associated with $\mathrm{dFdC}$ sensitivity (46). It was reported that a high CDA-to-dCK ratio may be a marker of resistance to decitabine (an analog of cytidine) (47). Hosokawa et al (42) identified high protein expression levels of CDA and low levels of dCK in their successfully established HCT116 cells resistant to decitabine and $\mathrm{dFdC}$. The protein expression levels of CDA and dCK were also examined in the present study, and similar results were obtained compared with those of a previous study (40), suggesting that the $\mathrm{dFdC}$-resistant TNBC cells, which were termed MDA-MB-231R cells, were successfully established.

The expression of wild-type Dm-dNK in cell lines leads to nuclear localization of the enzyme, which can be attributed to the presence of a nuclear localization signal at the C-terminal region of the protein (40). The nuclear import of the protein is abolished by the site-directed mutation of arginine-247 to serine, leading to a predominant cytosolic localization of the enzyme $(37,40)$. Our research team has previously investigated Dm-dNK for its potential application as a suicide gene; the results have revealed that wild-type Dm-dNK retains its activity when it is expressed in human cells, and it is localized to the nucleus, resulting in high cell sensitivity to several cytotoxic nucleoside analogs, including araT, araC, BVDU and $\mathrm{dFdC}(25,27,37,40)$. In the present study, either the wild-type nuclear Dm-dNK (dNK-GFP) or the cytosolic arginine-247 Dm-dNK mutant (dNKmut-GFP) was expressed using lentiviral vectors and the mutant cytosolic Dm-dNK was also demonstrated to possess highly similar levels of enzymatic activity and cytotoxicity compared with the wild-type dNK $(33,37)$, consistent with the findings of the present study. As one of the most effective substrates for Dm-dNK, $\mathrm{dFdC}$ exerts strong effects on Dm-dNK-expressing MDA-MB-231R cells, which exhibit a 50 -fold decrease in $\mathrm{IC}_{50}$ value for $\mathrm{dFdC}$ compared with that of untransfected cells (40), suggesting that the nucleoside analogs (prodrug)/Dm-dNK system may overcome drug resistance by lowering the $\mathrm{IC}_{50}$ values for these chemotherapeutic agents. As for the underlying mechanism, the apoptosis 

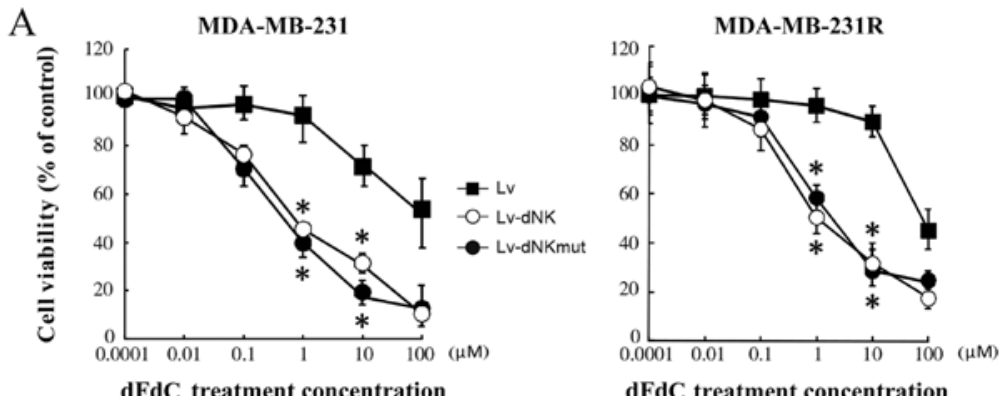

dFdC treatment concentration

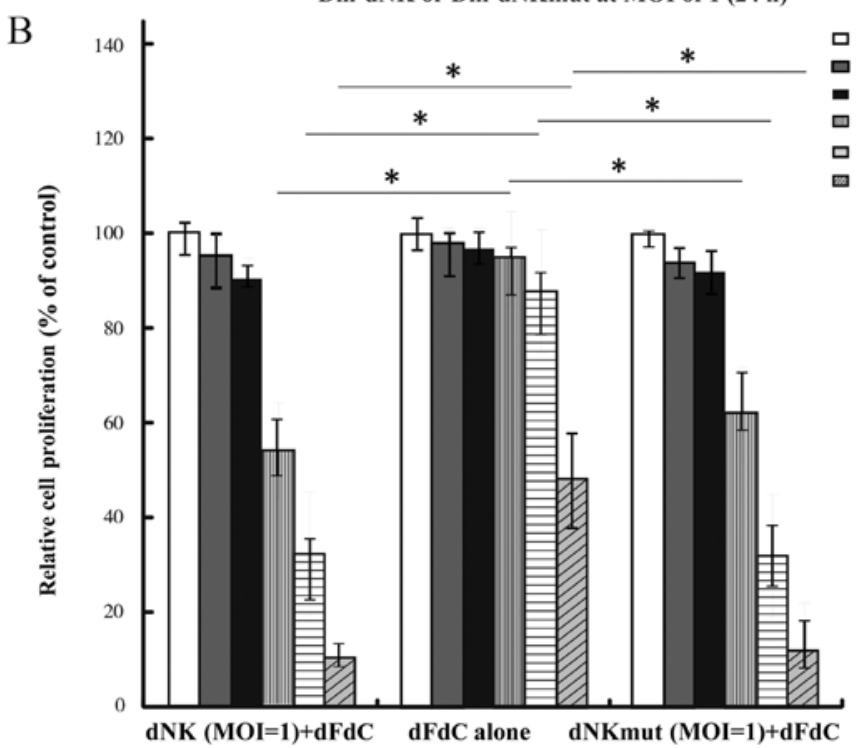

Figure 3. dNK and dNKmut reverse dFdC resistance in MDA-MB-231R cells. (A) The MDA-MB-231 and MDA-MB-231R cells were infected with Lv-dNK and Lv-dNKmut or lentiviral vector at an MOI of 1 and exposed to dFdC at various doses. MTT assay was used to determine the cell viability. (B) Cytotoxic effects of $\mathrm{dFdC}$ on MDA-MB-231R cells at different doses were determined by trypan blue dye exclusion assay. Cells transfected with lentiviral vector without $\mathrm{dFdC}$ were used as a control for $\mathrm{dNK}$ and $\mathrm{dNK}$ mut group. Untransduced cells without $\mathrm{dFdC}$ were used as a control for the $\mathrm{dFdC}$ alone group. ${ }^{*} \mathrm{P}<0.05$. MOI, multiplicity of infection; dFdC, gemcitabine; dNK, deoxyribonucleoside kinase; dNKmut, dNK mutant.
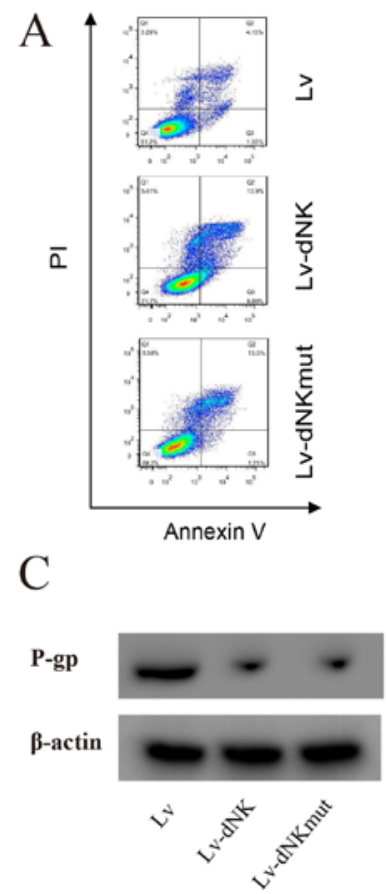

$\mathrm{B}$
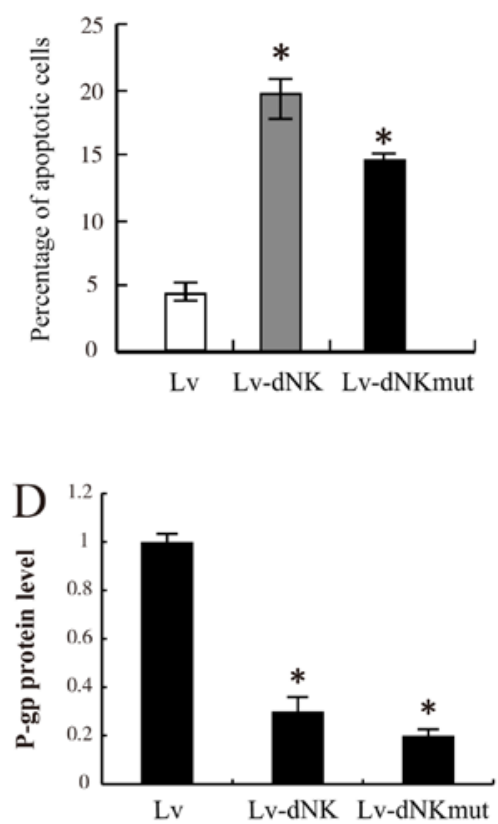

Figure 4.dNK and dNKmut may reverse dFdC resistance by increasing the apoptotic rate. (A and B) The levels of apoptosis in the transduced MDA-MB-231R cells were determined by flow cytometry. (C and D) Western blot analysis of P-gp expression in MDA-MB-231R cells transduced with or without dNK and dNKmut. $\beta$-actin was used as the loading control. " $\mathrm{P}<0.05$ vs. Lv. dFdC, gemcitabine; dNK, deoxyribonucleoside kinase; dNKmut, dNK mutant; P-gp, P-glycoprotein. 
assay revealed that such an increase in sensitivity may be attributed to an enhanced rate of apoptosis. To the best of the authors' knowledge, the present study was the first study to have examined the effects of the suicide gene Dm-dNK and its mutant on reversing drug resistance in TNBC cells.

The present study had certain limitations. Cancer cells that acquire resistance to one anticancer drug may also become simultaneously resistant to different drugs, which has been referred to as multidrug-resistance or cross-resistance $(42,48)$. Dm-dNK or Dm-dNKmut may also be able to reverse the drug-resistance that develops in cancer treatments with other chemotherapeutic agents, such as BVDU and araT, which was not investigated in the present study. In addition, the underlying mechanism of Dm-dNK reversing the drug resistance is still unclear and will be investigated in future studies.

In conclusion, Dm-dNK and Dm-dNKmut may be used to reverse the drug resistance encountered in cancer chemotherapy. This may form the basis for novel strategies in the treatment of patients with TNBC who have developed drug resistance.

\section{Acknowledgements}

Not applicable.

\section{Funding}

This work was supported by grants from the Natural Science Foundation of Liaoning Province (grant no. 20180551215), Key R \& D Plan Guidance Project of Liaoning Province (grant no. 2019JH8/10300020), and the Key Project of China Health Promotion Foundation (grant no. CHPF-RXO180301).

\section{Availability of data and materials}

The datasets used and/or analyzed during the current study are available from the corresponding author on reasonable request.

\section{Authors' contributions}

$\mathrm{YZ}$ and $\mathrm{XZ}$ designed the study. $\mathrm{YZ}, \mathrm{MG}$ and $\mathrm{CZ}$ performed the experiments. $\mathrm{YZ}$ and $\mathrm{HJ}$ collected and interpreted the data and performed the statistical analysis. YZ, HJ and XZ wrote and revised the manuscript. All authors read and approved the final manuscript.

\section{Ethics approval and consent to participate}

Not applicable.

\section{Patient consent to participate}

Not applicable.

\section{Competing interests}

The authors declare that they have no competing interests.

\section{References}

1. Peddi PF, Ellis MJ and Ma C: Molecular basis of triple negative breast cancer and implications for therapy. Int J Breast Cancer 2012: 217185, 2012
2. Thomas $\mathrm{H}$ and Coley HM: Overcoming multidrug resistance in cancer: An update on the clinical strategy of inhibiting p-glycoprotein. Cancer Control 10: 159-165, 2003.

3. Gonzalez-Angulo AM, Morales-Vasquez F and Hortobagyi GN: Overview of resistance to systemic therapy in patients with breast cancer. Adv Exp Med Biol 608: 1-22, 2007.

4. Cleator S, Heller W and Coombes RC: Triple-negative breast cancer: Therapeutic options. Lancet Oncol 8: 235-244, 2007.

5. Garrido-Castro AC, Lin NU and Polyak K: Insights into molecular classifications of triple-negative breast cancer: Improving patient selection for treatment. Cancer Discov 9: 176-198, 2019.

6. Li X, Lewis MT, Huang J, Gutierrez C, Osborne CK, Wu MF, Hilsenbeck SG, Pavlick A, Zhang X, Chamness GC, et al: Intrinsic resistance of tumorigenic breast cancer cells to chemotherapy. J Natl Cancer Inst 100: 672-679, 2008.

7. Rong D, Wang C, Zhang X, Wei Y, Zhang M, Liu D, Farhan H, Ali SA,Liu Y, Taouil A, et al: A novel taxane, difluorovinyl-ortataxel, effectively overcomes paclitaxel-resistance in breast cancer cells. Cancer Lett 491: 36-39, 2020.

8. Yuan D, Zhou H, Sun H, Tian R, Xia M, Sun L and Liu Y: Identification of key genes for guiding chemotherapeutic management in ovarian cancer using translational bioinformatics. Oncol Lett 20: 1345-1359, 2020.

9. Niero EL, Rocha-Sales B, Lauand C, Cortez BA, de Souza MM Rezende-Teixeira P, Urabayashi MS, Martens AA, Neves JH and Machado-Santelli GM: The multiple facets of drug resistance: One history, different approaches. J Exp Clin Cancer Res 33: 37, 2014.

10. Zhou M, Li L, Li L, Lin X, Wang F, Li Q and Huang Y: Overcoming chemotherapy resistance via simultaneous drug-efflux circumvention and mitochondrial targeting. Acta Pharm Sin B 9: 615-625, 2019.

11. Agarwal R and Kaye SB: Ovarian cancer: Strategies for overcoming resistance to chemotherapy. Nat Rev Cancer 3: 502-516, 2003.

12. Asleh K, Lyck Carstensen S, Tykjaer Jørgensen CL, Burugu S, Gao D, Won JR, Jensen MB, Balslev E, Laenkholm AV, Nielsen DL, et al: Basal biomarkers nestin and INPP4B predict gemcitabine benefit in metastatic breast cancer: Samples from the phase III SBG0102 clinical trial. Int J Cancer 144: 2578-2586, 2019.

13. Diéras V, Bonnefoi H, Alba E, Awada A, Coudert B, Pivot X, Gligorov J, Jager A, Zambelli S, Lindeman GJ, et al: Iniparib administered weekly or twice-weekly in combination with gemcitabine/carboplatin in patients with metastatic triple-negative breast cancer: A phase II randomized open-label study with pharmacokinetics. Breast Cancer Res Treat 177: 383-393, 2019.

14. Spratlin JL and Mackey JR: Human equilibrative nucleoside transporter 1 (hENT1) in pancreatic adenocarcinoma: Towards individualized treatment decisions. Cancers (Basel) 2: 2044-2054, 2010.

15. Hung SW, Marrache S, Cummins S, Bhutia YD, Mody H, Hooks SB, Dhar S and Govindarajan R: Defective hCNT1 transport contributes to gemcitabine chemoresistance in ovarian cancer subtypes: Overcoming transport defects using a nanoparticle approach. Cancer Lett 359: 233-240, 2015.

16. Honeywell RJ, Ruiz van Haperen VW, Veerman G, Smid K and Peters GJ: Inhibition of thymidylate synthase by 2',2'-difluoro-2'-deoxycytidine (Gemcitabine) and its metabolite 2',2'-difluoro-2'-deoxyuridine. Int J Biochem Cell Biol 60: 73-81, 2015.

17. Mitsuno M, Kitajima Y, Ohtaka K, Kai K, Hashiguchi K, Nakamura J, Hiraki M, Noshiro H and Miyazaki K: Tranilast strongly sensitizes pancreatic cancer cells to gemcitabine via decreasing protein expression of ribonucleotide reductase 1 . Int J Oncol 36: 341-349, 2010.

18. Bergman AM, Pinedo HM and Peters GJ: Determinants of resistance to 2',2'-difluorodeoxycytidine (gemcitabine). Drug Resist Updat 5: 19-33, 2002.

19. Baker JA, Wickremsinhe ER, Li CH, Oluyedun OA, Dantzig AH, Hall SD, Qian YW, Ring BJ, Wrighton SA and Guo Y: Pharmacogenomics of gemcitabine metabolism: Functional analysis of genetic variants in cytidine deaminase and deoxycytidine kinase. Drug Metab Dispos 41: 541-545, 2013.

20. Garcia-Diaz M, Murray MS, Kunkel TA and Chou KM: Interaction between DNA polymerase lambda and anticancer nucleoside analogs. J Biol Chem 285: 16874-16879, 2010.

21. Galmarini CM, Clarke ML, Santos CL, Jordheim L, Perigaud C, Gosselin G, Cros E, Mackey JR and Dumontet C: Sensitization of ara-C-resistant lymphoma cells by a pronucleotide analogue. Int J Cancer 107: 149-154, 2003. 
22. Réjiba S, Bigand C, Parmentier C and Hajri A: Gemcitabine-based chemogene therapy for pancreatic cancer using Ad-dCK::UMK GDEPT and TS/RR siRNA strategies. Neoplasia 11: 637-650, 2009.

23. Saiki Y, Yoshino Y, Fujimura H, Manabe T, Kudo Y, Shimada M, Mano N, Nakano T, Lee Y, Shimizu S, et al: DCK is frequently inactivated in acquired gemcitabine-resistant human cancer cells. Biochem Biophys Res Commun 421: 98-104, 2012.

24. Wang Q, Yang M, Zhang Y, Zhong L and Zheng X: Novel combination oncolytic adenoviral gene therapy armed with Dm-dNK and CD40L for breast cancer. Curr Gene Ther 19: 54-65, 2019.

25. Dong X, Qu W, Ma S, Zhu Z, Zheng C, He A, Karlsson A, $\mathrm{Xu} \mathrm{K}$ and Zheng X: Potent antitumoral effects of targeted promoter-driven oncolytic adenovirus armed with Dm-dNK for breast cancer in vitro and in vivo. Cancer Lett 328: 95-103, 2013

26. Jiang H, Zhao L, Dong X, He A, Zheng C, Johansson M, Karlsson A and Zheng X: Tanshinone IIA enhances bystander cell killing of cancer cells expressing Drosophila melanogaster deoxyribonucleoside kinase in nuclei and mitochondria. Oncol Rep 34: 1487-1493, 2015.

27. Ma S, Qu W, Mao L, Zhu Z, Jia L, Zhao L and Zheng X: Antitumor effects of oncolytic adenovirus armed with Drosophila melanogaster deoxyribonucleoside kinase in colorectal cancer. Oncol Rep 27: 1443-1450, 2012.

28. Qu W, Zhu Z, Zhao L, He A and Zheng X: Conditionally replicating adenovirus SG500-expressed mutant Dm-dNK gene for breast cancer therapy. Int J Oncol 41: 2175-2183, 2012

29. Tang M, Zu C, He A, Wang W, Chen B and Zheng X: Synergistic antitumor effect of adenovirus armed with Drosophila melanogaster deoxyribonucleoside kinase and nucleoside analogs for human breast carcinoma in vitro and in vivo. Drug Des Devel Ther 9: 3301-3312, 2015.

30. Zhang N,Dong X, Sun Y, Cai X,Zheng C,He A,Xu K and Zheng X: Cytotoxic effects of adenovirus- and lentivirus-mediated expression of Drosophila melanogaster deoxyribonucleoside kinase on Bcap37 breast cancer cells. Oncol Rep 29: 960-966, 2013.

31. Zhang N,Zhao L, Ma S, Gu M and Zheng X: Lentivirus-mediated expression of Drosophila melanogaster deoxyribonucleoside kinase driven by the hTERT promoter combined with gemcitabine: A potential strategy for cancer therapy. Int J Mol Med 30: 659-665, 2012

32. Zheng $X$, Lundberg $M$, Karlsson $A$ and Johansson $M$ : Lipid-mediated protein delivery of suicide nucleoside kinases. Cancer Res 63: 6909-6913, 2003.

33. Zhu Z, Ma S, Zhao L, Sun Z, He A, Xu H and Zheng X: Adenovirus-mediated Drosophila melanogaster deoxyribonucleoside kinase mutants combined with gemcitabine harbor a safe cancer treatment profile. Int J Oncol 38: 745-753, 2011.

34. Ma S, Zhao L, Zhu Z, Liu Q, Xu H, Johansson M, Karlsson A and Zheng X: The multisubstrate deoxyribonucleoside kinase of Drosophila melanogaster as a therapeutic suicide gene of breast cancer cells. J Gene Med 13: 305-311, 2011.

35. Sun X, Xu X, Chen Y, Guan R, Cheng T, Wang Y, Jin R, Song M and Hang T: Danggui buxue decoction sensitizes the response of non-small-cell lung cancer to gemcitabine via regulating deoxycytidine kinase and P-glycoprotein. Molecules 24: 2011, 2019.

36. Sierzega M, Pach R, Kulig P, Legutko J and Kulig J: Prognostic implications of expression profiling for gemcitabine-related genes (hENT1, dCK, RRM1, RRM2) in patients with resectable pancreatic adenocarcinoma receiving adjuvant chemotherapy. Pancreas 46: 684-689, 2017.
37. Zheng $\mathrm{X}$, Johansson $\mathrm{M}$ and Karlsson A: Nucleoside analog cytotoxicity and bystander cell killing of cancer cells expressing Drosophila melanogaster deoxyribonucleoside kinase in the nucleus or cytosol. Biochem Biophys Res Commun 289: 229-233, 2001.

38. Yang J,Wu Y,Wang X, Xu L,Zhao X and Yang Y: Chemoresistance is associated with overexpression of HAX-1, inhibition of which resensitizes drug-resistant breast cancer cells to chemotherapy. Tumour Biol 39: 1010428317692228, 2017.

39. Livak KJ and Schmittgen TD: Analysis of relative gene expression data using real-time quantitative PCR and the 2(-Delta Delta $\mathrm{C}(\mathrm{T})$ ) method. Methods 25: 402-408, 2001.

40. Zheng X, Johansson M and Karlsson A: Retroviral transduction of cancer cell lines with the gene encoding Drosophila melanogaster multisubstrate deoxyribonucleoside kinase. J Biol Chem 275: 39125-39129, 2000.

41. Fraser SP, Salvador V, Manning EA, Mizal J, Altun S, Raza M, Berridge RJ and Djamgoz MBA: Contribution of functional voltage-gated $\mathrm{Na}^{+}$channel expression to cell behaviors involved in the metastatic cascade in rat prostate cancer: I. Lateral motility. J Cell Physiol 195: 479-487, 2003.

42. Hosokawa M, Saito M, Nakano A, Iwashita S, Ishizaka A, Ueda K and Iwakawa S: Acquired resistance to decitabine and cross-resistance to gemcitabine during the long-term treatment of human HCT116 colorectal cancer cells with decitabine. Oncol Lett 10: 761-767, 2015.

43. Erin N, Grahovac J, Brozovic A and Efferth T: Tumor microenvironment and epithelial mesenchymal transition as targets to overcome tumor multidrug resistance. Drug Resist Updat 53: $100715,2020$.

44. Yang XL, Lin FJ, Guo YJ, Shao ZM and Ou ZL: Gemcitabine resistance in breast cancer cells regulated by PI3K/AKT-mediated cellular proliferation exerts negative feedback via the MEK/MAPK and mTOR pathways. Onco Targets Ther 7: 1033-1042, 2014

45. Triller N, Korosec P, Kern I, Kosnik M and Debeljak A: Multidrug resistance in small cell lung cancer: Expression of P-glycoprotein, multidrug resistance protein 1 and lung resistance protein in chemo-naive patients and in relapsed disease. Lung Cancer 54: 235-240, 2006.

46. Kroep JR, Loves WJ, van der Wilt CL, Alvarez E, Talianidis I, Boven E, Braakhuis BJM, van Groeningen CJ, Pinedo HM and Peters GJ: Pretreatment deoxycytidine kinase levels predict in vivo gemcitabine sensitivity. Mol Cancer Ther 1: 371-376, 2002.

47. Qin T, Castoro R, El Ahdab S, Jelinek J, Wang X, Si J, Shu J, He R, Zhang N, Chung W, et al: Mechanisms of resistance to decitabine in the myelodysplastic syndrome. PLoS One 6: e23372, 2011.

48. Stordal B, Pavlakis N and Davey R: Oxaliplatin for the treatment of cisplatin-resistant cancer: A systematic review. Cancer Treat Rev 33: 347-357, 2007.

This work is licensed under a Creative Commons Attribution-NonCommercial-NoDerivatives 4.0 International (CC BY-NC-ND 4.0) License. 